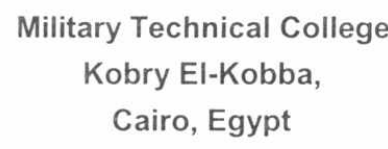

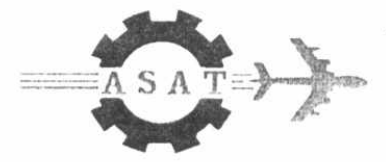

11-th International Conference

on Aerospace Sciences \&

Aviation Technology

\title{
DIRECT- SEQUENCE SPREAD SPECTRUM WITH DIVERSITY RECEPTION IN A SHADOWED NAKAGAMI FADING LAND MOBILE SATELLITE CHANNEL
}

\author{
Abdel-Hamid G.M. * and Abdel-Aziz A. M. *
}

\begin{abstract}
This paper is concerned with evaluating, the performance of a direct sequence spread-spectrum with two types of diversity copmbining techniques, namely, Selection diversity (SDC) and maximal ratio combining (MRC), in a land mobile satellite transmission system, using 16-ary Quadrature Amplitude modulation $\left(16^{\text {ary }} \mathrm{QAM}\right)$. It is assumed that the channel consists of a Nakagami shadowed lineof-sight signal plus Rayleigh distributed multipath signals, as well as additive white Gaussian noise. Fading and shadowing cause both envelope and phase variations of the received signal. The paper derives upper bounds of the probability of error due to both of these variations, and closed form expressions are found for L-branch diversity using both MRC and SDC in a realistic (practical) operating environments with independent but not identically distributed diversity paths. Assuming a Gaussian approximation for the interference, numerical results are obtained for both spread spectrum and spread spectrum with diversity eception in land mobile satellite communication systems with $16^{\text {ary }}$ QAM for light and average shadowing and fading
\end{abstract}

KEYWORDS: Land mobile satellite systems, Channel model, Nakagami model, Direct sequence spread-spectrum, Diversity combining techniques.

\footnotetext{
* Egyptian Armed Forces
} 


\section{INTRODUCTIO}

Land Mobile Satellite (LMS) systems are an important part of the third and fourth generation of wireless systems. The significance of such systems is rapidly growing for a variety of applications such as navigation, communications, broadcasting, etc. LMS systems provide services which are not feasible via land mobile terrestrial(LMT) systems. As a complement to LMT systems, LMS systems are able to serve many users over a wide area with low cost [1-2].

Spread spectrum techniques are well known and have been used successfully in military communication systems for several decades. Certain advantages, such as multipath mitigation and interference suppresion, make them a feasible alternative to existing multiuser mobile communication system. Spread spectrum multiple access systems are known to support more users than conventional time division multiple access ( TDMA) and frequency division multiple access (FDMA) systems. Direct sequence spread spectrum (DSSS) is usually preferred over other spread spectrum techniques because of its low implementation cost and because coherent modulation can be used in these systems. The CDMA system is a multiple access interference limited system, and its performance degrades as the number of users increases. Furthermore, the introduction of additional users into these systems only gives rise to very graceful performance degradation[3].

The bit error rate performance of each user in a CDMA system is also affected by the fading statistics of the received signal. A series of propagation experiments conducted in typical urban/suburban areas have revealed the multipath nature of the satellite mobile radio channel resulting from reflections, refractions, and scattering by buildings and other obstructions in the vicinity of the mobile. Multipath propagation can be resolved at the receiver of a direct-sequence code-division multiple-access (DS-CDMA) system that uses a bandwidth much larger than the coherence bandwidth of the channel. Empirical results as well as physical reasoning show that the total intensity of each resolvable path is formed by the superposition of radio waves that arrive at the receiver almost simultaneously. In this respect, the problem of obtaining the distribution of the signal strength of each path conincides with the random phasor interference problem. Where as, Rayleigh and Rician distributions are only special case solutions of the random vector problem, the Nakagami $m$ distribution provides a more general solution. This distribution is one of the most versatile, as it can model a variety of fading environments. Furthermore, Suzuki showed that paths with small delays beyond the LOS delay are better modeled by the Nakagami distribution.

In the spread-spectrum system, since the multipath propagation can be resolved at the receiver due to the wideband nature of the signal, the receiver can exploit the inherent multipath diversity characteristics of the channel to improve the signal reception. Coherent detection with diversity combining methods namely maximal ratio combining or selection diversity combining of the received paths is known to give best result in Additive White Gaussian Noise (AWGN) channels, when perfect channel estimation is possible. We assume that almost-perfect carrier phase recovery, channel phase, and gain estimation are feasible at the receiver side. In this case, a Rake receiver can take advantage of the multipath nature of the mobile channel to increase the Signal-to-Noise Ratio(SNR) of the output decision variable[4]. 
The performance analysis of a land mobile satellite communication system has been reported recently in [5\&6] using direct-sequence spread-spectrum with QPR and $16^{\text {ary }}$ QAM modulation. It is shown in [5\&6] that the spread-spectrum system yields better performance than the narrowband transmission if the line-of-sight path is dominant. Also, the performance analysis of $16^{\text {ary }}$ QAM with diversity reception in LMS channel has been reportd in [7], where we assumed independent and identically distributed(i.i.d) fading statistics. In this paper, we extendthe analysis presented in [7] in two fronts: First, we move away from the restriction of independent and identically distributed(i.i.d) fading statistics and study the effectiveness of MRC and SDC schemes in a more realistic (practical) operating environments. Due to the random nature of the propagation channel, it is more realistic to assume that the mean received signal strength and/or the fading severity index may be different for the different diversity paths. This may well be the case in an actual mobile link, scince the radio waves take different propagation paths and may undergo different amount of fading before arriving at the receiver. Secondary, we derive analytical expressions for evaluating the performance of MRC and SDC with direct sequence spread spectrum over LMS channel with independent but nonidentically distributed (i.n.d) diversity paths. Finally, the performance of MRC and SDC with DSSS are evaluated using measured parameters in various mobile environments for light and average shadowing and fading.

This paper is organized as follows: Section II summarizes the extended channel model and the receiver model. Section III deals with the performance analysis of the system using the two types of diversity techniques. The influence of the diversity techniques on the bit error probability is presented in section IV using computational results. Finally section $\mathrm{V}$ provides the conclusions.

\section{LAND-MOBILE SATELLITE SYSTEM MODEL}

This section is concerned with the channel model that will be used in the analysis. We shall use the shadowed Rice model given in [8] for a narrow-band channel in rural and suburban environments. The received signal is assumed to be the sum of a multipath signal with a Rayleigh distributed envelope and a shadowed LOS signal with Nakagami envelope distribution. The resulting probability distribution of the received signal envelope $r$ caused by the combined effect of fading and shadowing is given by [8]

$$
P_{\beta}(r)=\left(\frac{2 b_{0} m}{2 b_{0} m+\Omega}\right)^{m} \cdot \frac{r}{b_{0}} \cdot \exp \left(-\frac{r^{2}}{2 b_{0}}\right) \cdot 1, F_{1}\left(m, 1, \frac{\Omega r^{2}}{2 b_{0}\left(2 b_{0} m+\Omega\right)}\right), \quad r \geq 0
$$

where ${ }_{1} F_{1}(., .,$.$) is the confluent hypergeometric function, m$ is the Nakagami fading parameter (suited of propagation environment), $\Omega$ is the average power of the LOS component and $b_{0}$ is the average scattered power due to multipath.

The probability density function of the received signal phase $\Phi$ was found to be approximately Gaussian[9]

$$
P_{\phi}(\phi)=\frac{1}{\sqrt{2 \pi \sigma_{\phi}^{2}}} \cdot \exp \left(-\frac{\left(\phi-m_{\phi}\right)^{2}}{2 \sigma_{\phi}^{2}}\right)
$$


where $m_{\phi}$ and $\sigma_{\phi}^{2}$ are the mean and the variance of the received signal phase, respectively.

The above model is valid for a narrow-band system. If spread-spectrum modulation is used with chip duration of less than the delay spread of the channel, the multi-path power is partially reduced by the correlation operation in the receiver. The envelope and the phase distribution functions remain the same, but the values of $b_{0}$ and $\sigma_{\phi}$ are reduced. The impulse response of the wide-band channel can be written as:

$h(t)=\sum_{l=1}^{L} \beta_{l} \delta\left(t-\tau_{l}\right) \exp \left(j \phi_{l}\right)$

where $\beta_{l}, \tau_{l}$, and $\phi_{l}$ are the gain, time delay, and the phase of the $l^{\text {th }}$ path, respectively. The first path is the LOS and therefore (1) and (2) describe its propagation statistics. The other paths have a Rayleigh path gain distribution and uniformly distributed phase, since the direct LOS is suppressed by the correlation operation. The parameters of the various path distributions can be found if the power-delay profile is known. From measurements, it is known that the power delay profile can be modeled as [10]

$$
P(\tau)=\frac{1}{T_{m}} b_{o} \exp \left(-\frac{1}{T_{m}} \tau\right)
$$

where $T_{m}$ is the delay spread. For a rural environment, a typical value of $T_{m}$ is $0.65 \mu \mathrm{sec}[9]$. Due to the correlation operation, the multi-path power $b_{0}$ is reduced. For path $l$ it can be approximated as

$$
b_{l o}=b_{o}\left\{1-\exp \left(-\frac{T_{c}}{T_{m}}\right)\right\} \cdot \exp \left\{-(l-1) \frac{T_{c}}{T_{m}}\right\}
$$

where $T_{c}$ is the chip duration.

Also, the phase variance of the first path will be decreased, since it is determined by the amount of multi-path power and the statistics of the LOS propagation. Using (4.5.19) of [11], the phase distribution function $P_{\phi}(\phi)$ of a Nakagami shadowed Rician signal can be derived and given as[12]

$P_{\phi}(\phi)=\frac{1}{\sqrt{2 \pi}} \int_{0}^{\infty} e^{-z^{2} / 2 b_{o}}\left[1+G \sqrt{\pi} e^{G^{2}}[1+\operatorname{erf}(G)]\right] P(z) d z$

where $G \cong \frac{z \cos (\phi)}{\sqrt{2 b_{o}}}$, and $\operatorname{erf}($.$) is the error function: \operatorname{erf}(G) \cong \int_{0}^{G} e^{-t^{2}} d t$. It has been assumed that $\mathrm{P}(\mathrm{z})$ is Nakagami, given by[7] 


$$
P(z)=\frac{2 m^{m}}{\Gamma(m) \Omega^{m}} Z^{2 m-1} \cdot \operatorname{cxp}\left(-\frac{m Z^{2}}{\Omega}\right), Z \geq 0
$$

Where $\Gamma($.$) is the gamma function, m=\frac{\left(E\left[Z^{2}\right]\right)^{2}}{\operatorname{Var}\left(Z^{2}\right)} \geq 0$, is the Nakagami parameter with $\operatorname{Var}[$.$] , as the variance, and \Omega=E\left[Z^{2}\right]$ is the average power of the LOS component, hence

$$
P_{\phi}(\phi)=\frac{m^{m}}{\Gamma(m) \Omega^{m}} \int_{0}^{\infty} z^{2 m-1} \exp \left[\frac{-z^{2}}{2 b_{o}}-\frac{m \cdot z^{2}}{\Omega}\right]\left[1+G \sqrt{\pi} \exp \left(G^{2}\right)(1+\operatorname{erf}(G))\right] d z
$$

Now, the phase variance in the case of spread spectrum modulation can be determined by

$$
\sigma_{\phi}^{2}=\int_{-\pi}^{\pi} \phi^{2} \mathrm{P}(\phi) d \phi
$$

Note that the mean phase $\mu_{\phi}$ is zero because $P(\phi)=P(-\phi)$. Equation (8) and (9) are used only to determine the effect of spread-spectrum modulation on the phase variance. The combined effect of phase variation and envelope fading [8] is evaluated by using (2) as an approximation for (8). But, in the case of wide-band land-mobile satellite channel, the desired component of the received signal, is random due to random variations of the channel. It is clear that $X$ consists of amplitude $\beta$ with a shadowed Nakagami distributed, multiplied by the cosine of Gaussian distributed phase. Using [13\&14], the total distribution function of $x=\beta \cos \phi$ can be written as

$$
P_{X}(x)=2 \int_{|x|}^{\infty} P_{\beta}(r) P_{\phi}\left[\operatorname{arcos}\left(\frac{x}{r}\right)\right] \cdot \frac{d r}{\sqrt{r^{2}-x^{2}}}
$$

Substituting (1) and (2) in (10), the expression for probability density function of $x$ is

$$
\left.P_{X}(x)=\frac{2}{\sqrt{2 \pi \sigma^{2}}}\left(\frac{2 b_{0} m}{2 b_{0} m+\Omega}\right)^{m} \int_{t i l}^{\infty} \frac{r}{b_{0}} \exp \left[-I\left(\frac{r^{2}}{2 b_{0}}+\frac{a \cos ^{2}\left(\frac{x}{r}\right)}{2 \sigma_{\bullet}^{2}}\right)\right]\right]_{1} F_{1}\left(m, 1, \frac{\Omega r^{2}}{2 b_{0}\left(2 b_{0} m+\Omega\right)}\right) \frac{d r}{\sqrt{r^{2}-x^{2}}}
$$

equation (11) is valid in the case for which the receiver locks on the LOS signal. This requires the bandwidth of the carrier-tracking loop to be much smaller than the fading bandwidth of the received signal. If the bandwidth of the tracking loop is larger than the fading bandwidth, then the phase $\phi$ is approximately zero because the receiver will lock on the phase of the total signal. In the case, the distribution 
function $P_{x}(x)$ is equal to the shadowed Nakagami distributed $P_{\beta}(x)$. However, with a large tracking bandwidth the loop noise increases, so there is always a certain phase error. Therefore, in the bit error probability calculations one gets an upper bound by using (11) and a lower bound by using (1).

\section{DIVERSITY}

The idea of diversity is to combine several copies of transmitted signal, which undergo independent fading, to increase overall received power. So, when spreadspectrum modulation is used with a chip time that is less than the delay spread of the channel, a number of resolvable path $M$ exists that can be used to improve the performance. Two types of path diversity, namely maximal ratio combining and selection diversity are consider here. A description of these diversity techniques can be found in [13-15].

\section{A. Maximal Ratio Combininb (MRC)}

When maximal ratio combining is used, the received signal is coherently correlated with a particular code for $L$ different paths. Here $L$ is the order of diversity. Each path ( $\mathrm{i}^{\text {th }}$ path and user $\mathrm{K}$ ) is multipled by the instantananeous fading amplitude $\alpha_{i k}$ and all correlation outputs are combined. The probability density function of the sum of the squared path gains $a_{i k}{ }^{2}$ is the convolution of the $L$ different path gain probability density functions. Since the amplitude of the first path (LOS) with shadowing is characterized by Nakagami distribution given by (1), then the probability density function $P_{1}(x)$ of a squared of signal strength $\alpha_{i k}$ (shadowed LOS) given by a simple transformation $P_{1}(x)=\frac{P_{1}(\sqrt{x})}{2 \sqrt{x}}$, then

$$
P_{1}(x)=\left(\frac{m}{2 b_{0} m+\Omega}\right)^{m} \cdot \exp \left(-\frac{x}{2 b_{0}}\right) \cdot 1 F_{1}\left(m, 1, \frac{\Omega x}{2 b_{0}\left(2 b_{0} m+\Omega\right)}\right)
$$

All other paths are assumed to have uncorrelated independent but not identical Rayleigh distribution amplitudes with different average scattered power $\left(b_{i}\right)$, their gains have a Chi-square probability density function, so the sum of $\mathrm{L}-1$ different Chisquare variables result in the following probability density function

$$
P_{2}(x)=\frac{1}{2 b_{2}} \cdot \exp \left(\frac{-x}{2 b_{2}}\right) * \frac{1}{2 b_{3}} \cdot \exp \left(\frac{-x}{2 b_{3}}\right) \ldots * \frac{1}{2 b_{L}} \cdot \exp \left(\frac{-x}{2 b_{L}}\right)=\sum_{i=2}^{L} \frac{\left(2 b_{i}\right)^{L-3}}{\prod_{j=2 . j \neq i}^{L}\left(2 b_{i}-2 b_{j}\right)} \cdot \exp \left(\frac{-x)}{2 b_{i}}\right)(
$$

Where * denotes convolution. Now, the probability density function of the sum of all $L$ squared path gains can be obtained by a convolution of (12) and (13). 


$$
\begin{aligned}
& P_{m r c}(x)=P_{1}(x) * P_{2}(x)=\int_{0}^{x} P_{1}(x-\alpha) \cdot P_{2}(\alpha) d \alpha \\
& P_{m r c}(\alpha)=\frac{1}{2 b_{o}}\left(\frac{2 b_{0} m}{\left(2 b_{0} m+\Omega\right)}\right)^{m} \sum_{i=2}^{L} \frac{\left(2 b_{i}\right)^{L-3}}{\prod_{j=2, j+2}^{L}\left(2 b_{i}-2 b_{j}\right)_{0}^{x}} \exp \left(-\frac{\alpha}{2 b_{i}}+\frac{(x-\alpha)}{2 b_{0}}\right) \cdot F_{1}\left(m, 1, \frac{\Omega \cdot(x-\alpha)}{2 b_{0}\left(2 b_{0} m+\Omega\right)}\right) d \alpha
\end{aligned}
$$

where $\alpha=\sum_{i=1}^{L} \alpha_{i k}^{2} \quad$ gains of multipath received signal

\section{B. Selection Diversity Combining (SDC)}

With selection diversity, the strongest out of $L$ different signals is selected. The cumulative distribution function(cdf) of the output signal is the multiplication of all $\mathrm{L}$ different path gain cdf's, so the resulting pdf is

$P_{s d}(x)=\frac{d}{d x}\left[P_{1}(x) P_{2}(x) \ldots P_{i}(x) \ldots P_{L}(x)\right]$

Where $\quad P_{i}(x)=\left\{\begin{array}{cc}\text { shadowed } \text { Nakgami } & \text { for } \mathrm{i}=1 \\ \text { Rayleigh } & \text { otherwise }\end{array}\right.$, and

$P_{1}(x)=\int_{0}^{x} P_{r}(r) d r, \quad P_{i}(x)=1-\exp \left(-\frac{x^{2}}{2 b_{i}}\right), 1<\mathrm{i} \leq L$

Then the probability density function of the strongest out of $L$ different signals is $P_{s d}(x)=\frac{d}{d x}\left[\frac{1}{b_{0}} \cdot\left(\frac{2 b_{0} m}{2 b_{0} m+\Omega}\right)^{m} \int_{0}^{x} r \cdot \exp \left(\frac{-r^{2}}{2 b_{0}}\right),{ }_{1} F_{1}\left(m, 1, \frac{\Omega \mathrm{r}^{2}}{2 \mathrm{~b}_{0}\left(2 b_{0} m+\Omega\right)}\right) \prod_{i=2}^{L}\left(1-\exp \left(-\frac{x^{2}}{2 b_{i}}\right)\right) \cdot d r\right]$

\section{ERROR PROBABILITY ANALYSIS}

In this section, expressions for the average bit error rate (BER) for $16^{\text {ary }}$ QAM are derived. The average BER is defined as the number of erroneous bits per block of transmitted bits averaged over all time to acount for all channel fading variations. Thus, we are interested in the expected value of the BER, evaluated by averaging the conditional error probability of $16^{\text {ary }}$ QAM over the PDF of the combiner output, i.e. given by the integral over the range $0<r<\infty$

$\overline{P_{b}}(\gamma)=\int_{0}^{\infty} P_{b}(\gamma / x) \cdot P_{\operatorname{mrc}(s d c)}(x) d x$

where $\gamma$ is the SNR for the unfaded link given by $\gamma=E\left\{\left.X_{k}\right|^{2}\right\} / N_{0}=E_{b} / N_{0}, \mathrm{X}$ is the instantaneous signal power, $P_{b}(\gamma / x)$ is the instantaneous BER of $16^{\text {ary }}$ QAM signaling in an additive white Gaussian noise channel condioned on $X$, and has the upper bound given by [13]. 
$P_{b}(\gamma / x) \leq 4 Q\left[\sqrt{\frac{2 r^{2}}{5 \sigma^{2}}} \cos \phi\right],|\phi| \leq \pi$

A tie bound for the $Q($.$) in terms of the exponential function can be used [16], and$ hence, equation (18) can be put in the form

$$
P_{e} \leq 2 \exp \left[\frac{-r^{2}}{5 \sigma^{2}} \cos ^{2} \phi\right],|\phi| \leq \pi
$$

where $r$ is the root mean square amplitude of the received signal, $\sigma^{2}$ is the total variance of Gaussian noise

$$
\sigma^{2} \cong N_{o} T_{b}+\sigma_{\text {int }}^{2}
$$

$N_{0}$ is the noise power in bit-rate bandwidth, $T_{b}$ is the bit duration and $\sigma_{\text {int }}^{2}$ is the interference power, a closed form expression for the interference power is obtained as[12]

$$
\sigma_{\text {int }}^{2}=\frac{2 K A^{2} T_{h}^{2}}{3 N}\left[b_{o}+\frac{\Omega}{m}+\exp \left[\Psi(m)+\frac{\psi(m)}{2}\right]\right]
$$

where $A$ is the transmitted signal amplitude which is assumed to be constant and identical for all users, $\mathrm{K}$ is the total number of users, $\mathrm{N}$ is the length of the spreading code (assumed Gold code) which is assumed to be equal to $T_{b} / T_{c}$, and multipath power $b_{o}=\sum_{i=1}^{L} b_{i o}$, and $\psi(),. \psi^{\prime}(),. .$. are the psi function (the logarithmic derivative of the gamma function) and its derivatives, and $P_{\operatorname{mrc}}(x)$ is the Pdf of LMS shadowing and fading channel power given by (14). Substituting (14) and (19) into (17), the average BER for LMSC with MRC receiver can be written as

$$
P_{b}(\gamma)=\frac{1}{b_{0} \cdot \sqrt{2 \pi \sigma_{\phi}^{2}}}\left(\frac{2 b_{0} m}{\left(2 b_{0} m+\Omega\right)}\right)^{m} \cdot \sum_{i=2}^{L} \frac{\left(2 b_{i}\right)^{L-3}}{\prod_{i=2, i x j}^{L}\left(2 b_{i}-2 b_{j}\right)} \iint_{-\pi 0}^{\pi \infty x} \int_{0}^{\infty} \exp \left(-\left(\frac{x^{2} \cos \phi^{2}}{5 \sigma^{2}}+\frac{\alpha}{2 b_{i}}+\frac{\phi^{2}}{2 \sigma_{\phi}^{2}}+\frac{(x-\alpha)}{2 b_{0}}\right)\right), F_{1}\left(m, 1, \frac{\Omega(x-\alpha)}{2 b_{0}\left(2 b_{0} m+\Omega\right)}\right) d a d x d \phi
$$

By the same procedure. Substituting (16) and (19) into (17), the average BER for LMSC with SDC receiver can be written as

$P_{b}(\gamma)=\frac{2}{\sqrt{2 \pi \sigma_{\theta}^{2}}} \int_{-\pi 0}^{\pi x} \int_{0}\left[\exp \left(-\left(\frac{x^{2} \cos \phi^{2}}{5 \sigma^{2}}+\frac{\phi^{2}}{2 \sigma_{0}^{2}}\right)\right)\right] \cdot \frac{d}{d x}\left[\frac{1}{b_{0}} \cdot\left(\frac{2 b_{0} m}{2 b_{0} m+\Omega}\right)^{m} \int_{0}^{x} r \cdot \exp \left(\frac{-r^{2}}{2 b_{0}}\right) \prod_{i=2}^{L}\left(1-\exp \left(-\frac{x^{2}}{2 b_{i}}\right)\right),{ }_{1} F_{1}\left(m, 1, \frac{\Omega r^{2}}{2 b_{0}\left(2 b_{0} m+\Omega\right)}\right) d r\right] d x d \phi$

\section{NUMERICAL RESULTS}

In Table I the parameters from [7] are given with the new values for the multipath power $b_{0}$ in the $i^{\text {th }}$ path and phase variance $\sigma_{\phi}^{2}$, when spread-spectrum modulation is used. The results are obtained by numerically evaluating the probability of error expressions (21) and (22) derived in the pervious section, and compared for a bit rate $1 / T_{b}=2400 \mathrm{bit} / \mathrm{sec}$, code length $\mathrm{N}=4095$, chip time $T_{c}=T_{b} / N=0.1 \mu \mathrm{sec}$ and $\mathrm{K}=400$ users. The results are based on a per-branch signal-to-noise ratio $E_{b} / N_{0}$ used in 
the figures and defined as $E_{b} / N_{0}=A^{2} T_{b} / 2 \sigma^{2}$. (i.e., on average, $M R C$ with $L$ branches has $L$ times more signal energy than a single branch).

Table 1: channel Model Parameters

\begin{tabular}{|c|c|c|c|}
\hline & Light & Average & Heavy \\
\hline $\mathrm{m}$ & 19.4 & 10.1 & 0.739 \\
\hline$\Omega$ & 1.29 & 0.835 & $8.97 \times 10^{-4}$ \\
\hline $\mathrm{b}_{0}$ & 0.158 & 0.126 & 0.063 \\
\hline $\mathrm{b}_{1}$ & 0.023 & 0.018 & 0.009 \\
\hline$\sigma_{\phi}$ & 0,14 & 0.16 & 1.42 \\
\hline
\end{tabular}

Figure 1 shows the BER versus SNR for light shadowing and fading using MRC, with and without spreading. For the sake of comparison, BER is also plotted for the ideal $16^{\text {ary }}$ QAM case, i.e., $16^{\text {ary }}$ QAM without fading and shadowing. With no diversity (i.e., light shadowing and fading, L=1) and spreading, as expected, the performance of $16^{\text {ary }}$ QAM with light shadowing and fading was found to be worse than that of ideal $16^{\text {ary }}$ QAM. Thus, to achieve a desired BER at a reasonable SNR, some method of improving the performance is required. MRC with spreading is one such method. Comparing the diversity cases with spreading $(L=4)$ to the nondiversity case, we see that a significant increase in BER performance can be achieved through diversity with spreading, particularly, for an average BER for $10^{-4}$, an SNR improvement of 19 $\mathrm{dB}$, can be achieved over the nondiversity case, and $3 \mathrm{~dB}$ can be achieved over light shadowing and fading with spreading. For average shadowing and fading Fig. 2, the ratio of LOS power and multipath power is smaller and therefore the diversity gain is greater, about $4 \mathrm{~dB}$ for $\mathrm{P}_{\mathrm{e}}=10^{-4}$, and $\mathrm{L}=4$. Comparing Fig. 3 and 4 , it is seen that the spread spectrum modulation with SDC yields better performance than narrowband modulation and narrow-band with diversity.

Figure 5 and 6 comparing the diversity cases with spreading for different branches. Note that as the number of branches increases (diversity order increase), the incremental savings in SNR decreases. For satellite systems, where a single transmitter serves multiple environments, increasing the diversity order will reduce the excess signal power when the mobile is in a variable environments.

To show the effect of changes in the parameters used in the previous results, Figs. $7,8,9 \& 10$ depict the bit error probability for different values of $T_{c}$ and $N$, respectively. These figures are calculated for average shadowing and fading. The use of MRC and SDC with an order of diversity $(L=4)$. If one decreases the chip rate $T_{c}$ ( Fig. $7 \&$ 9), while the ratio $T_{b} / T_{c}=N$ is kept constant, the bit error probability decreases because of the increased multipath rejection. Adecrease of the code length $N$, while $T_{c}=T_{b} / N$ is fixed at 100 nsec, causes an increased bit error probability ( Fig 8 \& 10), because the interference power is inversely proportional to $\mathrm{N}$.

\section{CONCLUSIONS}

The bit error probability of $16^{\text {ary }} \mathrm{QAM}$ in combination with MRC, SDC and Direct sequence, have been evaluated in a more realistic (practical) operating 
environments for a land mobile satellite link in a rural and suburban environment with independent but not identically distributed (i.n.d) diversity path. The presence of fading, shadowing, and additive noise has been considered. The analysis includes both envolope and phase variations caused by fading and shadowing.

Using the measured channel parametera printed in [7], BER curves were ploted for various channel parameters. Without diversity reception, the BER performance of a $16^{\text {ary }}$ QAM was sufficiently poor in case of shadowing and fading environmenta, that the more bandwidth-efficient modulation schemes could not be utilized. However, it can be shown that the direct sequence spread spectrum with diversity reception provided significant improvements in the BER. Furthermore, increasing the number of diversity branches reduces the dependence of the average BER on fading and shadowing enviroments. Computional results further show that an increase in the number of users or a decrease of the code length for a fixed value of the chip time, decreases the performance of the system. However, an enhanced performance is obtained by decreasing the chip time for a fixed value of the code length. Thus there are two different ways to enhence the performance: first, the chip time can be decreased and second, the order of diversity can be increased. The choice between these two depends on bandwidth limitations and hardware considerations. A decreased chip time requires a larger bandwidth and faster hardware. A higher diversity order requires more hardware at the same speed.

As our results show, path diversity combining techniques with spreading seem to be very useful to improve the performance. In order to further enhance the performance, particularly in the case of heavy shadowing and fading, it is necessary to utilize coding techniques. The derived expressions are useful for the design of low-complexity receiver structures for wideband CDMA which ensuring graceful degradation of $A B E R$ performance with fewer rake fingers.

\section{REFERENCES}

[1] W.W.Wu., " Satellite communications", Proc. IEEE Trans. On Vech. Tech., Vol. 85, 1997, PP. 988-1010.

[2] J.V.Evans, "Satellite systems for personal communications", Proc. IEEE, Vol. 86, No. 4, 1998, PP. 1325-1341.

[3] K.S.Gilhousen et al., " On the capacity of a cellular CDMA system", IEEE Trans. On Vech. Tech., Vol. 46, No. 40, May 1991, PP. 303-312.

[4] George P. Efthymoglou, Valentine A. Aalo, Henry Helmken," Performance analysis of coherent DS-CDMA systems in a Nakagami fading channel with arbitrary parameters", IEEE Trans. On Vech. Tech., Vol. 46, No. 2, May 1997, PP. 289-297.

[5] Gamal Mabrouk Abdel Hamid "Quadrature Partial Response Direct Sequence Spread Spectrum for Land Mobile Satellite Communicatio", Nineteenth National Radio Science Conference, March 19-21, 2002, Alexandria University, Egypt.

[6] Gamal Mabrouk Abdel-Hamid, El-Sayed A. Gadallah. And Salah El-Agooz “ Analysis of 16-ary Quadrature Amplitude Modulation Direct-Sequence Spread Spectrum for Land - Mobile Satelite Communication Channels", $3^{\text {rd }}$ ICEENG 
Conference on Electrical Engineering, May 2002, 14-16, Military Technical College-Egypt.

[7] Gamal Mabrouk Abdel Hamid, "Performance Analysis of 16-ary Quadrature Amplitude Modulation with Diversity Reception for Land - Mobile Satelite Channel", $21^{\text {th }}$ National Radio Science Conference (NRSC 2004), March 16-18, 2004, NTI- Egypt.

[8] A Abdi, H.Allen Barger, and M. Kaveh, "A simple Alternative to the lognormal model for shadow fading in terrestrial and satellite channels ", Proc. IEEE Vech. Tech. Conf., Atlantic city, NJ, 2001, PP. 2058-2062.

[9] C.Loo, "Measuements and Models of a Land Mobile Satelite Channel and their applications to MSK", IEEE Trans. Vech. Tech., Vol. 36, August 1987, PP. 114121.

[10] J. Van Rees, "Measrements of the wide band radio channel characteristics for rural, residential and suburban areas", IEEE Trans. on Vehicular Technol., Vol. VT-36, PP. 2-6, February 1987.

[11] P. Backman, Probability in communication engineering, New York: Harcourt, Brace \& World, 1967.

[12] Richard D. J. Van Nee, Howard S. Misser and Ramjee Prasad, "DirectSequence Spread Spectrum in a Shadowed Rician Fading Land-Mobile Satellite Channel", IEEE Journal. on Selected Areas in Comm., Vol. 10, PP. 350-357, February 1992.

[13] J.G. Proakis, Digital communications, New york, McGraw-Hill, 1989.

[14] W.C.Jakes, Ed., "Microwave Mobile Communications" New York, Wiely, 1974.

[15] W.C.Y. Lee, "Mobile Communication Engineering", New York, McGraw-Hill, 1982.

[16] J.M. Wozencraft and I.M.Jacobs, "Principles of Communication Engineering ", New-York, Wiely, 1985, PP. 83-84.

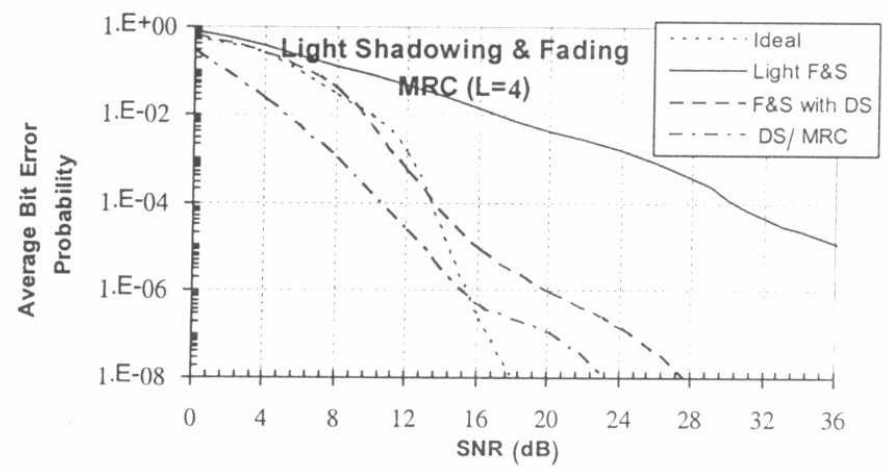

Fig.1. Bit error probability for light shadowing \& fading using MRC, with $k=400$ users, chip length $T_{c}=0.1 \mu \mathrm{sec}$, Gold code length $\mathrm{N}=4095$, and bit rate $r_{b}=2400 \mathrm{~b} / \mathrm{s}$. 


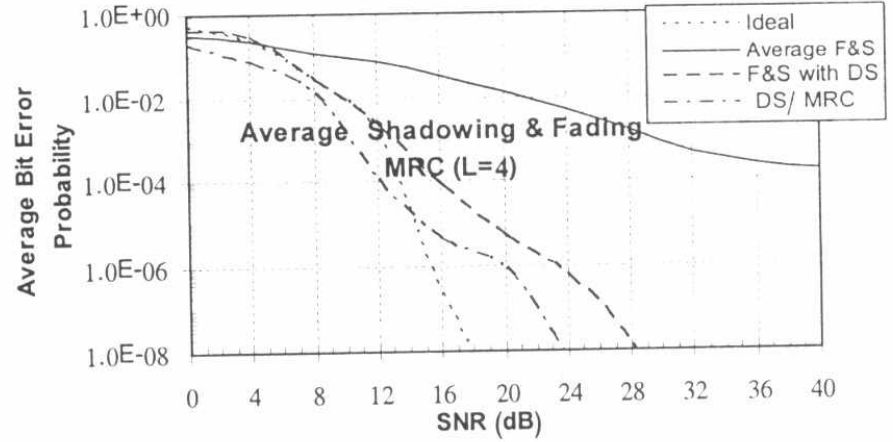

Fig.2. Bit error probability for Average shadowing \& fading using MRC, with $\mathrm{k}=400$ user, chip length $T_{c}=0.1 \mu \mathrm{sec}$, Gold code length $\mathrm{N}=4095$, and bit rate $r_{b}=2400 \mathrm{~b} / \mathrm{s}$.

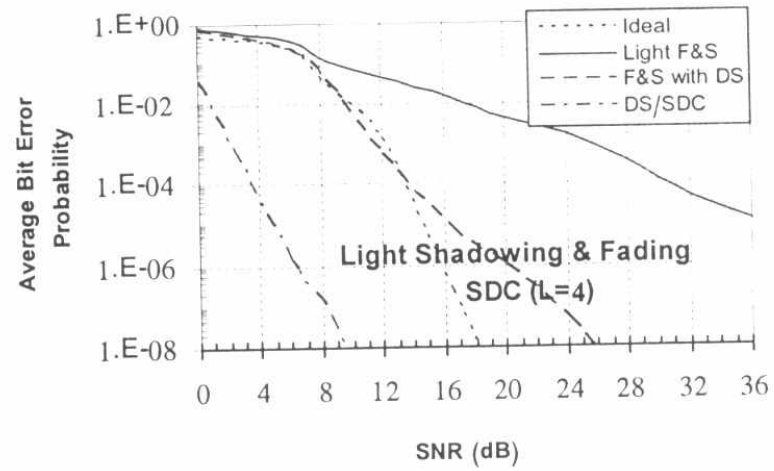

Fig.3. Bit error probability for light shadowing \& fading using SDC, with $k=400$ user, chip length $T_{c}=0.1 \mu \mathrm{sec}$, Gold code length $\mathrm{N}=4095$, and bit rate $r_{b}=2400 \mathrm{~b} / \mathrm{s}$.

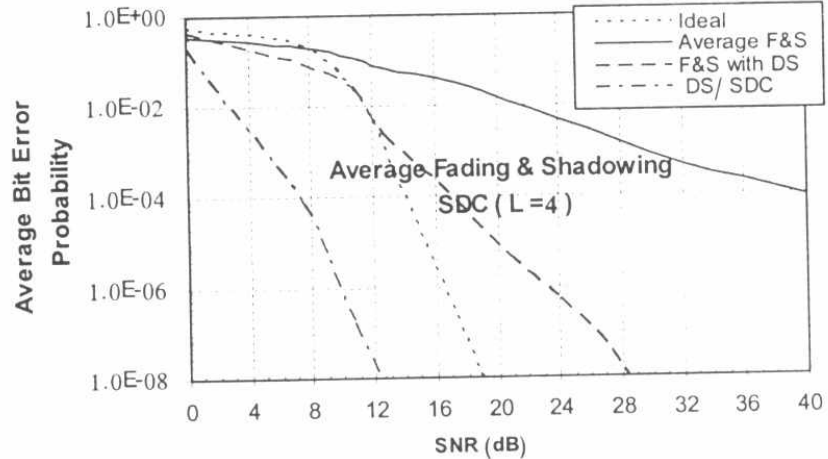

Fig.4. Bit error probability for Average shadowing \& fading using SDC, with $\mathrm{k}=400$ user, chip length $T_{c}=0.1 \mu \mathrm{sec}$, Gold code length $\mathrm{N}=4095$, and bit rate $r_{b}=2400 b / s$. 


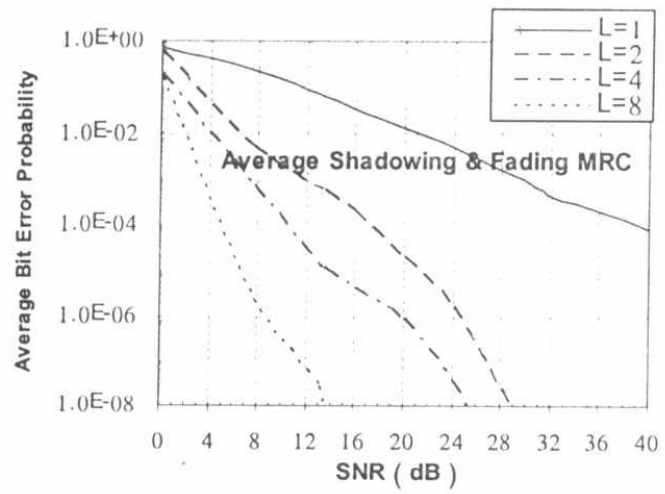

Fig.5. Bit error probability for Average shadowing \& fading using MRC, for different paths, with $\mathrm{k}=400 \mathrm{user}$, chip length $T_{c}=0.1 \mu \mathrm{sec}$, Gold code length $\mathrm{N}=4095$, and bit rate $r_{b}=2400 \mathrm{~b} / \mathrm{s}$.

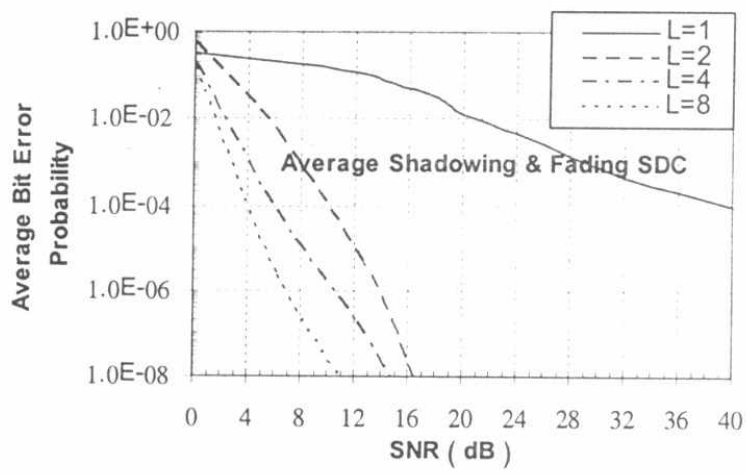

Fig. 6. Bit error probability for Average shadowing \& fading using SDC, for different paths, with $\mathrm{k}=400$ user, chip length $T_{c}=0.1 \mu \mathrm{sec}$, Gold code length $\mathrm{N}=4095$, and bit rate $r_{b}=2400 b / s$.

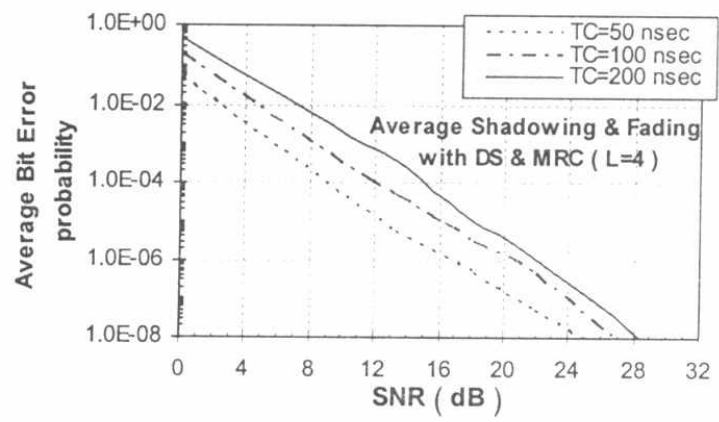

Fig.7. Bit error probability for Average shadowing \& fading using MRC, with $k=400$ user, Gold code length $\mathrm{N}=4095$, bit rate $r_{b}=2400 \mathrm{~b} / \mathrm{s}$, and $T_{c}$ as parameter. 


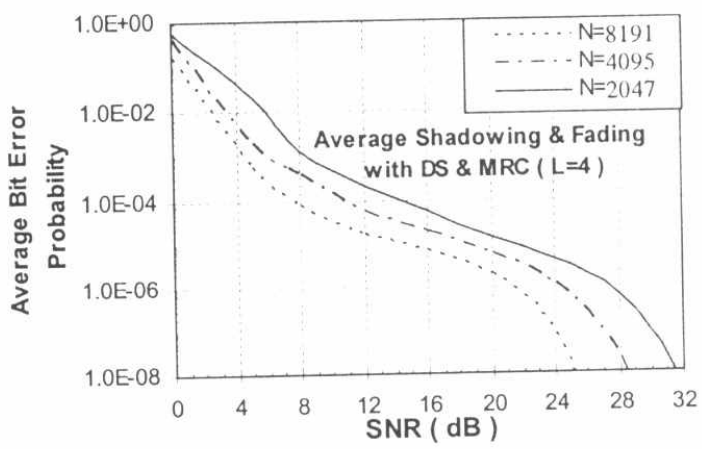

Fig.8. Bit error probability for Average shadowing \& fading using MRC, with $k=400$ user, chip length $T_{c}=0.1 \mu \mathrm{sec}$, bit rate $r_{b}=2400 b / s$, and $\mathrm{N}$ as parameter.

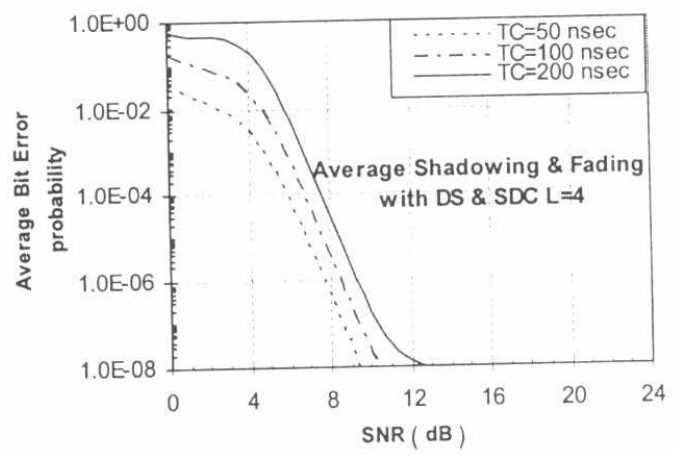

Fig.9. Bit error probability for Average shadowing \& fading using SDC, with $k=400$ user, Gold code length $\mathrm{N}=4095$, bit rate $r_{b}=2400 \mathrm{~b} / \mathrm{s}$, and $T_{c}$ as parameter.

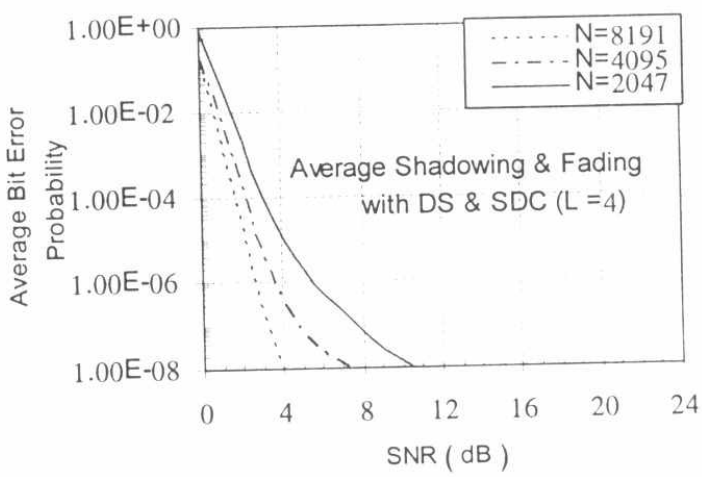

Fig.10. Bit error probability for Average shadowing \& fading using SDC, with $k=400$ user, chip length $T_{c}=0.1 \mu \mathrm{sec}$, bit rate $r_{b}=2400 \mathrm{~b} / \mathrm{s}$, and $\mathrm{N}$ as parameter. 